\title{
El planeamiento como instrumento para la protección de los conjuntos históricos
}

Juan Manuel Becerra García

Arquitecto

Jefe del Servicio de Protección de la Dirección General de Bienes Culturales

Desde que la técnica jurídica en materia de patrimonio histórico identificara una tipología específica para la protección de las ciudades históricas con valores patrimoniales relevantes, los Conjuntos Históricos, se hizo necesario dotarla de un régimen tutelar propio que permitiera adaptarse a las características de una realidad tan compleja.

De esta forma, aún durante la vigencia de la Ley de 13 de mayo de 1933, sobre Defensa, Conservación y Acrecentamiento del Patrimonio Histórico Español, se redactan en los ajos sesenta las llamadas Instrucciones para la defensa de los Conjuntos Histórico-Artísticos que pretenden superar la dimensión proteccionista y estática de las medidas e instrumentos articulados en la norma republicana, y paliar de alguna manera la desconexión con los instrumentos urbanísticos que la reciente Ley del Suelo de 1956 nos había dotado como medio de regular los procesos de crecimiento y transformación de las ciudades.

Es el afianzamiento de la técnica urbanística como instrumento para modelar el paisaje tanto urbano como no urbano, y en definitiva para definir la capacidad de intervención y transformación del medio natural o antropizado, lo que hace que cuando se redacta la ley 16/1985, de 25 de junio, del Patrimonio Histórico Español, se confíe en esta técnica como el principal instrumento sobre el que descansar la protección de los Conjuntos Históricos, Zonas Arqueológicas y Sitios Históricos, aunque el legislador le dedica un mayor desarrollo articulado a la primera de la figuras que a las demás.

Por otra parte, al remitir la protección de los Conjuntos Históricos a una figura de planeamiento, se lograba otro efecto largamente anhelado: evitar la inseguridad que para toda actuación suponía la doble autorización administrativa. Por una parte, el procedimiento reglado de la licencia municipal sustentado en un documento público como es el plan urbanístico, y de otra, la autorización vinculante de las Comisiones del Patrimonio Histórico, órganos colegiados de expertos que dictaminan sobre la bondad de las intervenciones en el momento de proponerse éstas.
Pero, ¿qué se les pide a los planes que desarrollan la protección de los Conjuntos Históricos?

Aunque algunas veces se los han tachado de planes "fachadistas" al mostrar en la practica una atención especial a aspectos relacionados con la imagen exterior de las edificaciones y al ambiente de sus calles y plazas. En realidad, desde un primer momento, tanto la legislación del patrimonio histórico autonómica como la estatal, se decantan por una tipología de planes de protección que entre sus determinaciones contemplen aspectos tales como el mantenimiento de las tramas urbanas, de las tipologías arquitectónicas, el uso residencial o el de las actividades tradiciones.

La legislación del Patrimonio Histórico Andaluz nos remite a unas Directrices para la formulación de planeamiento municipal que son aprobadas por la Consejería de Cultura una vez comunicado por los Ayuntamientos la voluntad de inicio del procedimiento de elaboración de planeamiento de protección. El legislador ha dejando libertad a la administración para que perfile y concrete el contenido de las mismas, limitándose a establecer que incidirán sobre aspectos relacionados con la conservación, protección y potenciación del Patrimonio Histórico, así como que pueden orientar sobre la figura de planeamiento a utilizar para dar cumplimiento a la obligación prevista en el artículo 32 de la Ley I/199|' del PHA. En otro lugar, la Ley establece que

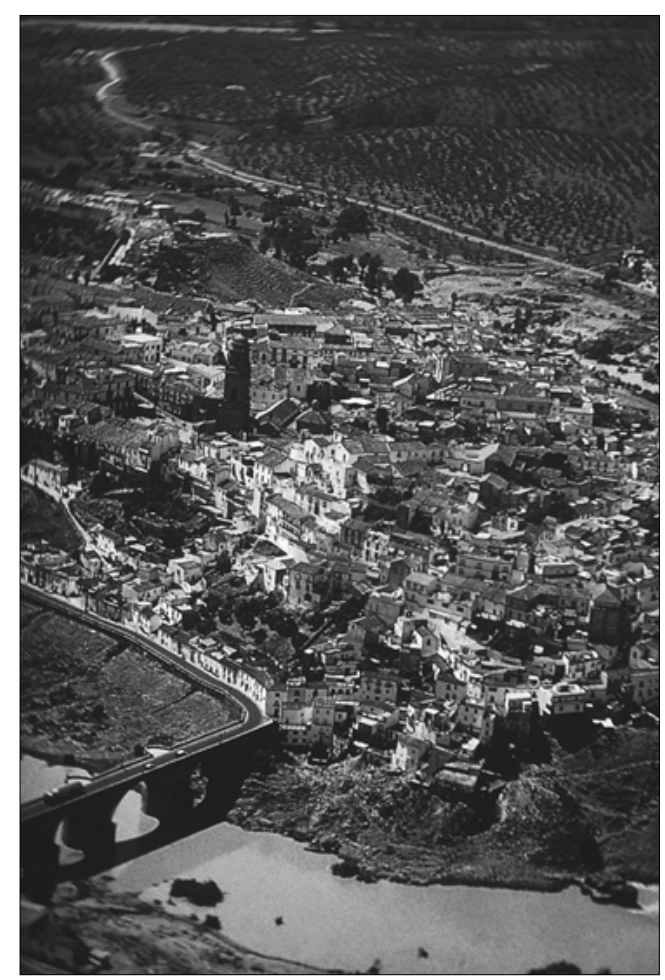

Montoro, Córdoba. Municipio de gran riqueza espacial y monumental. Posee uno de los primeros Planes Especiales de Protección del Conjunto Histórico redactados en Andalucía. En la actualidad ha creado, con apoyo de la administración autonómica, una oficina municipal para la gestión y ejecución del Plan Especial. 


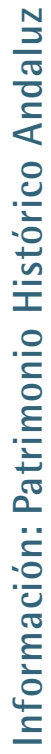

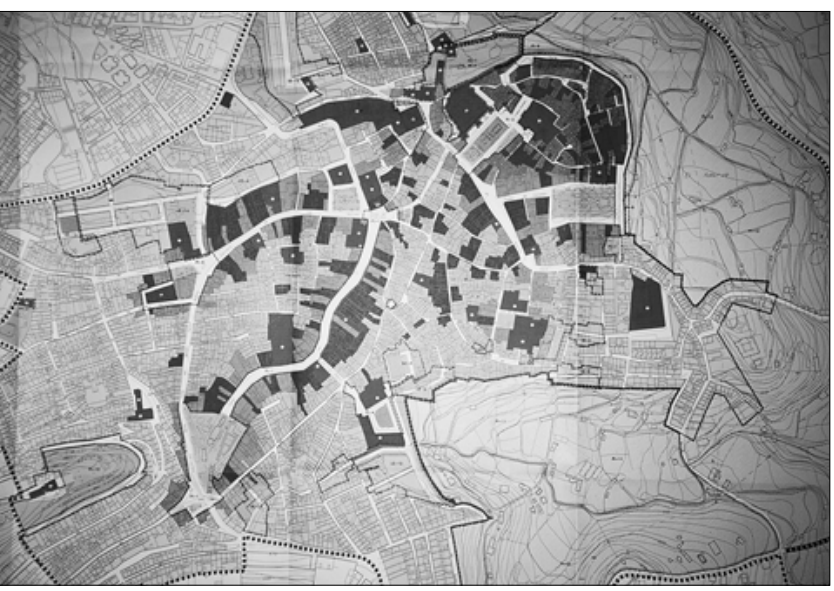

Priego de Córdoba. Municipio de tamaño medio en donde el

Conjunto Histórico ha quedado descentrado respecto al crecimiento de la ciudad actual. El Plan Especial aprobado supera los límites del $\mathrm{CH}$ para incluir las áreas de borde. en la formación, modificación o revisión del planeamiento, se señalarán los criterios para la determinación de los elementos tipológicos básicos de las construcciones, y de la estructura o morfología urbana que deban ser objeto de potenciación o conservación.

Por otro lado, la Ley 16/1985 del PHE hace un desarrollo más pormenorizado de los contenidos de los planes de protección mostrando, entre otras determinaciones, una gran preocupación por el mantenimiento de la estructura urbana y arquitectónica de los Conjuntos Históricos, así como de las características generales de su ambiente, considerando excepcionales las sustituciones de inmuebles aunque sean parciales, y estableciendo que, en todo caso, se deben mantener las alineaciones urbanas existentes. También considera excepcionales las remodelaciones urbanas, justificándolas sólo en el caso de que impliquen una mejora en su relación con el entorno territorial o urbano o que eviten usos degradantes para el Conjunto Histórico. En otro momento alude a la recuperación de las áreas residenciales y de las actividades económicas adecuadas cuando promueve la delimitación de posibles áreas de rehabilitación integrada.

Por último, el Catálogo Urbanístico se reconoce como el complemento inseparable de un planeamiento de protección, al permitir de forma individualizada para los elemento con valor del Conjunto Histórico, tanto inmuebles edificados como espacios libres, estructuras significativas o componentes naturales que lo acompañen, definir el tipo de intervención posible en función del interés o valor que el elemento posea.

En definitiva, no solo debe ser objetivo de los planes de protección el mantenimiento de unas formas y espacios heredados, sino que también deben establecer medidas para el mantenimiento y potenciación de los usos, actividades y relaciones que son consustanciales con la definición de cada Conjunto Histórico.

De esta forma, la formalización del plan de protección en un Conjunto Histórico estará determinado por los valores identificados en el mismo. O lo que es lo mismo, la suma y la ponderación de los valores existentes en un Conjunto Histórico determina las características y concreción del planeamiento de protección: valores monumentales, arquitectónicos, morfológicos, tipológicos, ambientales, de perspectiva, de uso y actividad, de fiestas y tradiciones, de ubicación y entorno, de relación y movilidad, valores de superposición (entornos, arqueología subyacente y emergente), etc. ${ }^{2}$

En concreto, cuando la administración cultural de la Junta de Andalucía subvenciona la redacción de planes especiales de protección en Conjuntos Históricos les exige como objetivos que deben alcanzarse los siguientes:

a) De investigación arqueológica e histórica que enriquezca el conocimiento de la ciudad y permita su transmisión y difusión entre la población. Se deben identificar las distintas zonas arqueológicas conocidas y localizadas; las áreas en las que se presume la existencia de restos arqueológicos; los límites de la ciudad antigua y su evolución; los trazados urbanísticos, tramas históricas, ensanches que se reconozcan en la ciudad y que hayan aportado alguna significación cultural a la formación de la misma, los monumentos, conjuntos y jardines históricos, etc.

b) Asegurar la integración del Conjunto Histórico con los nuevos crecimientos y aumentar el grado de legibilidad de su estructura urbana, a través de un adecuado tratamiento y mejora de las condiciones de borde y de percepción del conjunto, puntos de referencia, visuales y perfiles urbanos, operaciones de remodelación y mejora de la trama urbana que sean precisas, etc.

c) Establecimiento de medidas para la protección de los valores paisajísticos existentes: las vistas, fachadas y perfiles urbanos que desde el exterior ofrece el propio núcleo histórico.

d) Mantenimiento de las características de la trama urbana, de las alineaciones y de las tipologías parcelarias y edificatorias.

e) Especial atención a la recuperación y tratamiento adecuado de los espacios libres existentes (calles, plazas,...) o con posibilidades de mejora mediante su reordenación.

Se marcan las directrices de diseño urbano de los espacios libres y se incide sobre los aspectos de pavimentación, mobiliario urbano, arbolado, ajardinamiento, etc; es decir, sobre la construcción de los espacios libres.

f) Ordenación de la estructura viaria de forma que se garantice la accesibilidad para las actividades urbanas y se resuelvan los conflictos de tráfico y necesidades de aparcamiento. Se definirá la sección transversal de las vías que requieran una nueva configuración y vías de nuevo trazado.

g) Mejora de las condiciones de vida de las áreas residenciales, a través de actuaciones sobre sus condiciones de habitabilidad y la mejora de las dotacio- 
nes de equipamiento, servicios, accesibilidad e infraestructuras básicas, instrumentando las medidas necesarias para potenciar la rehabilitación del patrimonio residencial, fomentando la permanencia de la población residente y captación de nuevos habitantes.

h) En orden a la operatividad del Plan Especial, se deberán establecer los cauces de coordinación de las Administraciones con competencias en el Conjunto, fomentando los posibles acuerdos con las distintas Administraciones para ordenar las inversiones públicas de acuerdo con los objetivos del Plan, orientando dichas intervenciones públicas dada su capacidad de actuar como regeneradores urbanos.

i) Proteger y poner de manifiesto los valores históricos y formales de espacios urbanos, edificios y demás elementos significativos, a través de una rigurosa catalogación, que se corresponda con unos tipos definidos de actuación, de forma que se garantice el mantenimiento de las estructuras y tipologías tradicionales, así como la contención de los procesos de renovación y deterioro, determinando su capacidad arquitectónica para asumir nuevos usos y capacidad de transformación. El Catálogo deberá compatibilizarse con la Ley del Patrimonio Histórico en relación a la identificación de Bienes de Interés Cultural y sus entornos o de los del Catálogo General del Patrimonio Histórico de Andalucía.

j) Establecer las condiciones a que se han de someter las edificaciones de nueva planta y las sustituciones de edificios no catalogados para conseguir su integración ambiental y morfológica en el conjunto histórico.

En realidad estos objetivos pueden cumplirse tanto en planes especiales de protección como en planeamiento de carácter general: planes generales de ordenación urbana o normas subsidiarias de planeamiento municipal con contenido de protección. La elección de una figura u otra de planeamiento para la materialización de la protección depende de las características tipológicas del Conjunto Histórico, que se suelen asociar con el tamaño poblacional de la ciudad donde se encuentre enclavado.

De esta manera, para los Conjuntos Históricos de pequeños municipios, con población menor de 10.000 habitantes, que suelen estar constituidos por núcleos compactos normalmente en asentamientos singulares de origen defensivo y en donde el Conjunto Histórico coincide con la totalidad del núcleo urbano, con fuertes valores ambientales y paisajísticos, se suele redactar normas subsidiarias de planeamiento municipal con contenido de protección.

En cambio, los Conjuntos Históricos de ciudades medias, con poblaciones entre 10.000 y 50.000 habitantes, con núcleos de cierto desarrollo urbano y en donde el centro histórico suele empezar a perder actividad y centralidad, con una arquitectura monumental que convive con la popular, y con una gran calidad en la escena y ambiente urbano, se suele optar por la redacción de planes especiales de protección del Conjunto Histórico, planes que suelen incluir en su ámbito algo más que el Conjunto Histórico estricto para establecer determinaciones sobre las áreas de charnela con el resto de la ciudad.

En los Conjuntos Históricos de los grandes municipios, con poblaciones superiores a los 50.000 habitantes, el Conjunto Histórico es solo una parte de la ciudad, con problemas de interrelación con ella, bordes con falta de definición, áreas pendiente de rehabilitar, degradación en la escena urbana, insuficiencia del sistema de comunicaciones, equipamientos y espacios libres, en estos casos se recomienda la división en áreas menores el Conjunto Histórico para poder abordarlo con la figura más adecuada, normalmente la redacción de planes especiales de protección y reforma interior ${ }^{3}$.

Pero evidentemente, cualquier técnico de la administración reconoce que el mejor de los planes de protección de un Conjunto Histórico apenas garantiza el mantenimiento y conservación del Conjunto Históri-

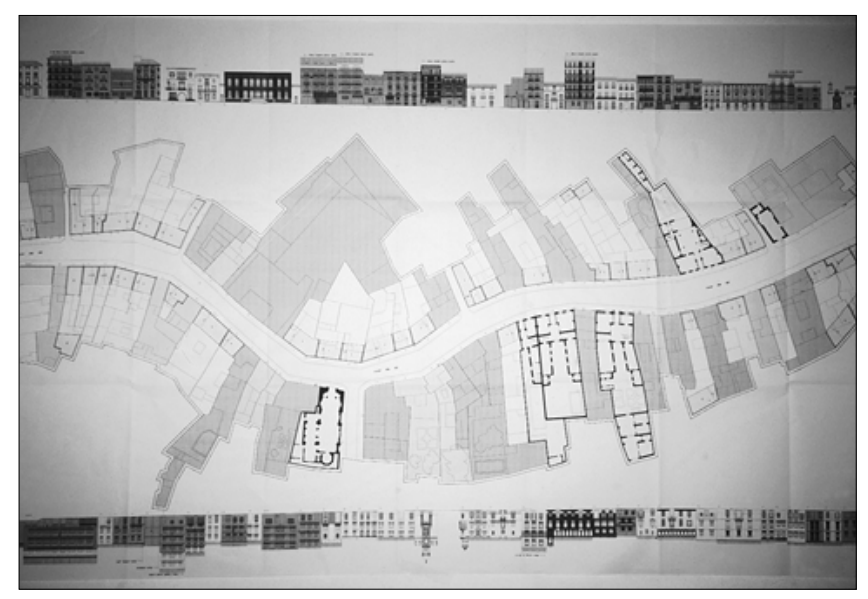

co, si no viene acompasado de otra serie de medidas que lo apoyen. Estamos cansados de ver planes con magníficos desarrollos normativos, catálogos urbanísticos completísimos, y propuestas de actuación brillantes, cuyo Talón de Aquiles es la escasa capacidad de gestión urbanística de los Ayuntamientos, y a los que la administración de Cultura acaba de transferir la capacidad de autorización en base al plan aprobado. $\bigcirc$ planes especiales de protección que se ven incapaces de evitar la terciarización del centro histórico, al ser el modelo inducido desde la ordenación superior del planeamiento general. $\bigcirc$ áreas que se degradan paulatinamente a pesar de las medidas de choque propuestas por el planeamiento, al no poderse acoger suficientemente los propietarios o inquilinos a los incentivos para su rehabilitación.

Es necesario pues, una vez aprobados los planes de protección, un programa de actuaciones e inversiones con números reales que sirva de base para un compromiso entre las administraciones actuantes en favor
Priego de Córdoba. Detalle de las ordenanzas gráficas en la Calle Río. 


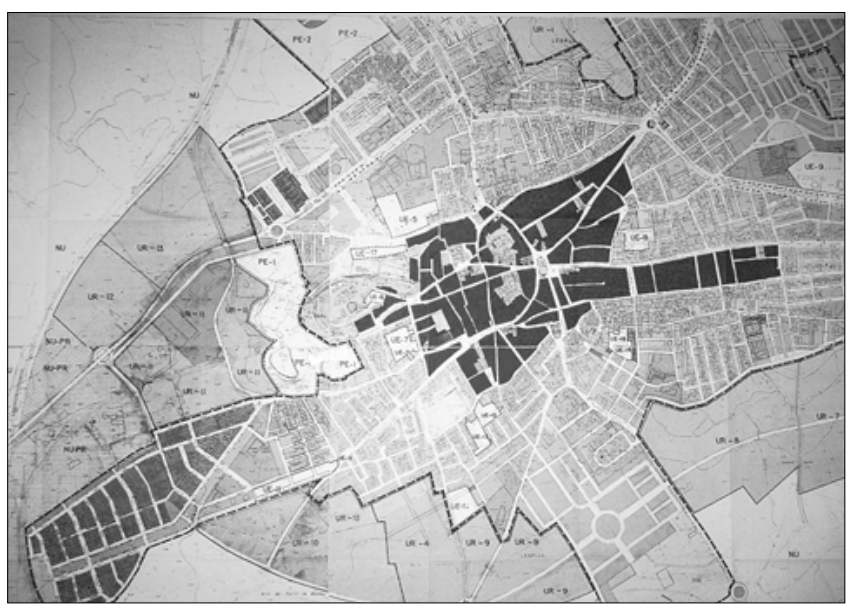

Lebrija, Sevilla. Municipio en donde el Conjunto Histórico -zona oscuraes solo una pequeña parte del suelo urbano, y el Plan General de Ordenación Urbana en redacción es el que va a contener los contenidos de protección. de la conservación del Conjunto Histórico, y que permita por un lado dotar a los Ayuntamientos que lo precisen ${ }^{4}$, de unos técnicos cualificados, y por otro, garantías de inversión suficiente para ejecutar las políticas de protección y conservación previstas en los planes.

Cada vez estamos más convencidos de que la coordinación entre las administraciones y la valoración de su patrimonio por los ciudadanos, son la base para la tutela efectiva de los Conjuntos Históricos.
Un paso adelante, del que aún hay mucho que seguir impulsando, lo supuso el acuerdo entre dos departamentos de la Junta de Andalucía para aunar esfuerzos en favor de la protección y conservación de los Conjuntos Históricos de tamaño pequeño y medio de la Comunidad Autónoma. El Programa Regional de Planeamiento en Centros Históricos aprobado en 1994 por las Consejerías de Cultura y de Obras Públicas y Transportes, pretende establecer con los Ayuntamiento acogido al Programa, un marco general de cooperación de apoyo a la redacción de planeamiento de protección, de acuerdo con la exigencia establecida por la legislación del patrimonio histórico, así como a la gestión y ejecución de dicho planeamiento.

En la actualidad casi la mitad de los casi I 30 Conjuntos Históricos con que cuenta Andalucía han redactado o están redactando su planeamiento de protección. Unos acogidos al Programa de Centros Históricos y otros por iniciativa de la Consejería de Cultura, o municipal. Entre los municipios que ya cuentan con planeamiento de protección aprobado definitivamente, se encuentran Almería (PGOU), Vélez Rubio (NNSS), Cádiz (PGOU), Puerto Real (PECH), Montoro (PECH), Priego de Córdoba $(\mathrm{PECH})$, Alhambra-Alijares (PECH), Albaicín (PECH), y diversos sectores del Conjunto Histórico de Sevilla (PECH).

\section{Notas}

I. Recordemos que la LPHE, en su artículo 20, establece la obligación para todo Conjunto Histórico de dotarse de un Plan Especial de Protección del área afectada por la declaración u otro instrumento de planeamiento que cumpla en todo caso con las exigencias establecidas en la Ley. Y que el artículo 32 de la LPHA concreta que los instrumentos de planeamiento podrán ser los siguientes:

a) Planes Especiales de Protección o de Reforma Interior.

b) Planes Generales de Ordenación Urbana.

c) Normas Subsidiarias o Complementarias de Planeamiento de ámbito municipal.

d) Planes Parciales.

e) Cualquier otro instrumento de planeamiento que se cree por la legislación urbanística, siempre que cumpla funciones equivalentes a los anteriores enumerados.

2. Evidentemente estamos hablando de espacios vivos, en los que se desarrolla la actividad humana, y no de ciudades museo que se activan con el primer turista que llega por la mañana y se apagan con el último bar que cierra por la noche. En este caso el plan de protección tendría otros componentes que lo sustentasen con prioridad de los aspectos formales o ambientales y de accesibilidad.
3. Un mayor desarrollo de la problemática asociada a cada tipología de Conjunto Histórico según el tamaño poblacional se encuentra publicada en el Cuaderno IX del IAPH Indicadores para la evaluación del estado de conservación de Ciudades Históricas, en el artículo "Sobre la Conservación de los Centros Históricos en Andalucía" del autor.

4. Hay que recordar que casi 40 de los Conjuntos Históricos en Andalucía corresponden a municipios con población menor de 2.000 habitantes, en donde si hay arquitecto municipal éste se encuentra compartido con otros municipios. Por supuesto su capacidad de inversión es mínima. Otros 40 Conjuntos Históricos, corresponden a municipios con población comprendida entre 2.000 y 10.000 habitantes, en los cuales la situación apenas difiere del caso anterior. 